\title{
Massgeschneiderte Fortbildung
}

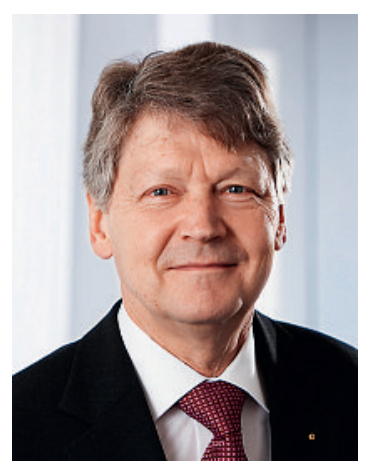

Die Schweizer Ärzteschaft bildet sich regelmässig fort. Seit dem 1. September 2007, Datum der Inkraftsetzung des Medizinalberufegesetzes (MedBG), gehört die Fortbildung zu den Berufspflichten aller in der Schweiz tätigen Ärztinnen und Ärzte. Wer das Fortbildungsprogramm einer Fachgesellschaft absolviert hat, erhält ein entsprechendes Diplom, mit dem sich die Ärztinnen und Ärzte u.a. gegenüber Patienten, Behörden und Versicherern ausweisen können. In dieser Ausgabe der Schweizerischen Ärztezeitung zeigt Christoph Hänggeli, Ge- einzelnen Fortbildungsprogrammen festgehalten. Ein allgemeinverbindlicher, detaillierter Lernzielkatalog für die Fortbildung würde zu einer massiven unkontrollierbaren Überreglementierung führen.

Das von den Fachgesellschaften zusammen mit dem SIWF ausgestellte Fortbildungsdiplom ist den Mitgliedern der FMH vorbehalten. Nichtmitglieder erhalten eine gleichwertige Bestätigung.

Die individuelle Kontrolle des Fortbildungsprotokolls ist Aufgabe der Fachgesellschaften. Rund 97 Prozent der Ärztinnen und Ärzte erfüllen gemäss den Rückmeldungen der Fachgesellschaften die Vorgaben des jeweiligen Fortbildungsprogramms und damit die geforderten 150 Credits innerhalb dreier Jahre. Ein Ausbau der Kontrolle durch ein summatives Assessment von einzelnen Kenntnissen oder Fähigkeiten, wie dies in unterschiedlichem Umfang in Grossbritannien und

\section{Das SIWF hat zur Erleichterung des administrativen Aufwands bei der Ausstellung von Fortbildungsdiplomen die Schaffung eines webbasierten Fortbildungsportals eingeleitet}

schäftsführer des SIWF, auf, welchen Stellenwert und welchen Nutzen das Fortbildungsdiplom für Ärztinnen und Ärzte hat [1]: Das Fortbildungsdiplom dient einerseits als Nachweis für die gesetzlich geforderte Fortbildungspflicht und andererseits für die Abrechnungsberechtigung gemäss TARMED.

\section{Das Fortbildungsdiplom dient einerseits als Nachweis für die gesetzlich geforderte Fort- bildungspflicht und andererseits für die Ab- rechnungsberechtigung gemäss TARMED}

Die Fortbildungspflichtigen sollen ihren Fortbildungsbedarf selbst bestimmen gemäss ihrer aktuellen Berufstätigkeit. Daraus leiten sich Mittel, Inhalte und Methodik der Fortbildung ab. Das Auflisten einzelner Fähigkeiten in der Durchführung von Untersuchungen oder Eingriffen ist in diesem Kontext nicht möglich; der zeitliche Umfang ist jeweils in den in den Staaten Nordamerikas erfolgt [2], wird von uns wie in allen Anrainerstaaten abgelehnt. Hingegen diskutiert die SIWF-Arbeitsgruppe «Unterstützung der Fachgesellschaften in der Fortbildung» ein formatives Assessment im Rahmen eines individuellen Fortbildungsportfolios. Das SIWF hat zur Erleichterung des administrativen Aufwands bei der Ausstellung von Fortbildungsdiplomen die Schaffung eines webbasierten Fortbildungsportals eingeleitet [3]. So können in Zukunft Ärztinnen und Ärzte ohne grossen Aufwand die erfüllte Fortbildung mit dem Diplom vorzeigen.

Dr. med. Max Giger, Präsident SIWF

\section{Literatur}

1 Hänggeli C. Besitzen Sie ein Fortbildungsdiplom? Schweiz Ärztezeitung. 2009;90(49):1906-8.

2 Shaw K, Cassel CK et al. Shared Medical Regulation in a Time of Increasing Calls for Accountability and Transparency. JAMA. 2009;302:2008-14.

3 Giger M, Hänggeli C. SIWF - die wichtigsten Projekte. Schweiz Ärztezeitung. 2009;90(26/27):1034-36. 